\title{
Metodología para la elaboración de la Guía de práctica clínica para el diagnóstico y el tratamiento de la pubertad precoz
}

\author{
Margarita Torres-Tamayo ${ }^{1}$, Jessie N. Zurita-Cruz², Blanca E. Aguilar-Herrera ${ }^{3}$, América L. Miranda-Lora ${ }^{4}$, \\ Raúl Calzada-León ${ }^{5}$, Aleida J. Rivera-Hernández², Marco A. Morales-Pérez ${ }^{6}$, Miriam M. Padrón-Martínez ${ }^{5}$, \\ María L. Ruiz-Reyes ${ }^{5}$, Leticia M. García-Morales ${ }^{4}$, Consuelo Barrón-Uribe ${ }^{3}$, Sletza L. Arguinzoniz-Valenzuela ${ }^{5}$, \\ Mayra C. Torres-Castañeda7, Lorena Lizárraga-Paulin7, Jorge A. Núñez-Hernández ${ }^{8,9}$, \\ Judith Cornejo-Barrera ${ }^{10}$, María T. Vidal-González ${ }^{11}$, María R. Martínez-Alvarado ${ }^{1}$, Elisa Nishimura-Meguro ${ }^{3}$, \\ Luz E. Bravo-Ríos ${ }^{3}$, Eulalia P. Garrido-Magaña², José A. Orozco-Morales ${ }^{4}$, Patricia G. Medina-Bravo ${ }^{4}$, \\ Ninel Coyote-Estrada ${ }^{3}$ y M. Fernanda Castilla-Peón ${ }^{4 *}$
}

${ }^{1}$ Instituto Nacional de Cardiología Ignacio Chávez, Ciudad de México; ${ }^{2}$ Hospital de Pediatría, Centro Médico Nacional Siglo XXI, Instituto Mexicano del Seguro Social (IMSS), Ciudad de México; ${ }^{3}$ Sociedad Mexicana de Endocrinología Pediátrica; ${ }^{4}$ Hospital Infantil de México Federico Gómez, Ciudad de México; Instituto Nacional de Pediatría, Ciudad de México; ${ }^{6} \mathrm{Hospital} \mathrm{General} \mathrm{de} \mathrm{Zona} \mathrm{No.} \mathrm{18,} \mathrm{IMSS,} \mathrm{Playa} \mathrm{del} \mathrm{Carmen,} \mathrm{Quintana} \mathrm{Roo;}$ ${ }^{7}$ Hospital General, Centro Médico Nacional La Raza, IMSS, Ciudad de México; ${ }^{8}$ Hospital General Regional 220, IMSS, Estado de México; ${ }^{9}$ Instituto Materno Infantil del Estado de México, Toluca, Estado de México; ${ }^{10} \mathrm{Hospital}$ Infantil de Tamaulipas, Ciudad Victoria, Tamaulipas; ${ }^{11} \mathrm{Hospital}$ del Niño y el Adolescente Morelense, Cuernavaca, Morelos. México

\begin{abstract}
Resumen
Introducción: La Sociedad Mexicana de Endocrinología Pediátrica presenta recomendaciones para el diagnóstico y el tratamiento de la pubertad precoz (PP), condición definida como el desarrollo de caracteres sexuales por incremento en la secreción hipofisiaria de gonadotropinas antes de los 8 años en las niñas y de los 9 años en los niños. Métodos: Se realizaron tres revisiones sistemáticas de ensayos clínicos controlados sobre intervenciones para el tratamiento de la PP, pruebas diagnósticas y estudios observacionales sobre efectos a largo plazo de la PP. La evaluación de la calidad de los estudios y la extracción de datos se realizó por pares. La evidencia se graduó con el sistema de la Scottish Intercollegiate Guidelines Network (SIGN) y del Oxford Centre for Evidence-Based Medicine (OCEBM) para las recomendaciones sobre la intervención y el diagnóstico, respectivamente. Las recomendaciones generadas se sometieron a un consenso por el método Delphi y fueron validadas por otros 143 endocrinólogos pediatras certificados mediante un cuestionario en línea. Resultados: Mediante consenso se generaron 12 recomendaciones para el diagnóstico de PP, siete sobre diagnóstico de causas secundarias de $P P$, ocho sobre intervenciones para inhibición de la pubertad, cinco sobre otras intervenciones en PP y 14 para la monitorización del tratamiento y el seguimiento de estos pacientes. Se obtuvo más del $90 \%$ de aprobación para cada una de las recomendaciones por el grupo de endocrinólogos certificados que respondieron el cuestionario en línea. Conclusiones: Si bien se logró un alto grado de consenso para las recomendaciones para el diagnóstico, el tratamiento y la monitorización de la PP entre los endocrinólogos pediatras, el nivel de evidencia para la mayoría de estas recomendaciones resultó bajo.
\end{abstract}

Palabras clave: Pubertad precoz. Análogos de la hormona liberadora de gonadotropina. Diagnóstico. Tratamiento. Guía de práctica clínica.

Correspondencia:

*M. Fernanda Castilla-Peón

E-mail: fernandacastillapeon@gmail.com
Disponible en internet: 23-06-2020 Bol Med Hosp Infant Mex. 2020;77(Supl 1):1-6

www.bmhim.com 1665-1146/@ 2020 Hospital Infantil de México Federico Gómez. Publicado por Permanyer. Este es un artículo open access bajo la licencia CC BY-NC-ND (http://creativecommons.org/licenses/by-nc-nd/4.0/). 


\section{Methodology for the development of the Clinical guideline for the diagnosis and treatment of precocious puberty}

\section{Abstract}

Background: The Mexican Society of Pediatric Endocrinology presents recommendations for the diagnosis and treatment of precocious puberty (PP), a condition defined as the development of sexual characteristics due to an increase in pituitary gonadotropin secretion before 8 or 9 years of age in girls and boys, respectively. Methods: Three systematic reviews were conducted: controlled clinical trials on interventions for PP treatment, diagnostic tests, and observational studies on the longterm effects of PP. The quality evaluation and data extraction from the studies were conducted by two independent reviewers. The Scottish Intercollegiate Guidelines Network and the Oxford Center for Evidence-Based Medicine systems were used for grading the quality of evidence for recommendations on intervention and diagnosis, respectively. Recommendations were submitted to a consensus by a Delphi method and were validated by another 143 certified pediatric endocrinologists through an online questionnaire. Results: The group generated 12 recommendations on the diagnosis of PP, seven on the diagnosis of secondary causes of PP, eight on interventions for inhibition of puberty, five on other interventions for PP treatment, and 14 for the monitoring and follow-up of these patients. The online questionnaires submitted to certified pediatric endocrinologists showed more than $90 \%$ of approval for each one of the recommendations. Conclusions: Although a high degree of consensus for the recommendations for diagnosis, treatment, and monitoring of PP among pediatric endocrinologists was achieved, most of these recommendations showed a low level of evidence.

Key words: Precocious puberty. Gonadotropin-releasing hormone analogs. Diagnosis. Treatment. Clinical practice guideline.

\section{Introducción}

La pubertad precoz (PP) de origen central es el desarrollo de caracteres sexuales secundarios - como consecuencia de la activación del eje hipotálamo -hipófisis-gónada - a una edad cronológica menor que la observada en la población general, lo que potencialmente tiene consecuencias indeseadas para la salud y el bienestar integral del paciente.

No hay un punto de corte universalmente aceptado para considerar la pubertad como precoz, ya que la asociación entre una edad específica de desarrollo y las "consecuencias indeseadas para la salud» es compleja. En muchas de las poblaciones estudiadas, alrededor del $95 \%$ de los sujetos inician la pubertad después de los 8 años en el sexo femenino y de los 9 años en el masculino, por lo que, en general, se considera el inicio del abordaje diagnóstico -y posiblemente terapéutico- para evitar consecuencias indeseadas de la pubertad en sujetos que la inician antes de estas edades ${ }^{1-3}$.

Es importante considerar lo siguiente:

- En algunas poblaciones, sobre todo de etnia no caucásica, la pubertad ocurre a edades más tempranas con mayor frecuencia.

- El inicio de la pubertad antes de los 8 o 9 años no necesariamente presenta consecuencias negativas para la salud.

Tomando en cuenta las consideraciones anteriores, y para fines de esta guía de práctica clínica (GPC), la pubertad precoz se define como el desarrollo de caracteres sexuales secundarios como consecuencia de la activación del eje hipotálamo-hipófisis-gónada antes de los 8 años en las niñas y antes de los 9 años en los niños.

Dentro de los riesgos potenciales o de las situaciones no deseadas asociados con la PP se encuentran:

- Condiciones que se manifiestan como PP y que ponen en riesgo la vida o la función del paciente (por ejemplo, neoplasias, hiperplasia suprarrenal congénita, entre otros).

- Posible impacto en la talla final.

- Impacto en la salud mental del paciente y en su calidad de vida a corto, mediano y largo plazo.

- Impacto a largo plazo en desenlaces en las áreas cardiometabólica, de fertilidad y de calidad de vida. - Impacto económico del abordaje diagnóstico y terapéutico.

En México existe variabilidad en el manejo de los pacientes con PP entre los distintos centros que los atienden. La variabilidad principalmente se relaciona con el abordaje diagnóstico, las indicaciones para iniciar y suspender el bloqueo con análogos de la hormona liberadora de gonadotropina $(\mathrm{aGnRH})$ y el tipo de seguimiento que se debe dar a estos pacientes.

El propósito de esta GPC es orientar a los proveedores de salud que atienden niños y adolescentes a identificar casos probables de PP, así como a médicos especialistas en endocrinología en el diagnóstico, el tratamiento y el seguimiento de estos pacientes. 
Las recomendaciones de esta GPC son específicas para pacientes en quienes se sospecha una etiología de origen central como causa del desarrollo de los caracteres sexuales; es decir, para niños con un volumen testicular $>4 \mathrm{~cm}^{3}$ antes de los 9 años y para niñas con desarrollo mamario antes de los 8 años. No incluye recomendaciones sobre casos de precocidad sexual de origen periférico, por lo que no hace recomendaciones sobre pacientes de sexo masculino con caracteres sexuales cuyo volumen testicular es $<4 \mathrm{~cm}^{3}$ ni para niñas en quienes se haya descartado la secreción prematura de gonadotropinas.

Esta GPC tampoco hará recomendaciones sobre el diagnóstico ni el manejo de condiciones clínicas distintas a la PP, excepto al considerarlas como diagnósticos diferenciales. No se harán recomendaciones sobre intervenciones en sujetos que se encuentren durante el periodo de pubertad si esta no inició antes de los 8 años en las niñas o de los 9 años en los niños.

Esta GPC pretende incidir en los siguientes aspectos:

- La identificación eficiente de causas secundarias de PP.

- La disminución de la morbilidad psiquiátrica y la mejoría de la calidad de vida en casos de niños, adolescentes y adultos que hayan presentado PP.

- La disminución de eventos adversos relacionados con el uso de aGnRH para el bloqueo de la pubertad.

- La optimización de otros desenlaces en salud a corto, mediano y largo plazo relacionados con la PP.

\section{Métodos para el desarrollo de la Guía de práctica clínica}

\section{Conformación del grupo desarrollador de la GPC}

El grupo estuvo conformado por 24 miembros titulares de la Sociedad Mexicana de Endocrinología Pediátrica con capacitación en evaluación de la literatura científica y un asesor metodológico.

\section{Conflicto de intereses}

Para el desarrollo de esta GPC se recibió apoyo de los laboratorios Asofarma - relacionado con la logística de tres reuniones del grupo de trabajo-, para la obtención de material bibliográfico y para el apoyo metodológico.
Tabla 1. Niveles de evidencia para estudios sobre intervenciones terapéuticas o profilácticas de acuerdo con SIGN (Scottish Intercollegiate Guidelines Network) ${ }^{4}$

\begin{tabular}{|l|l|}
\hline $1++$ & $\begin{array}{l}\text { Metaanálisis y revisiones sistemáticas de alta calidad } \\
\text { de ensayos clínicos con muy poco riesgo de sesgo }\end{array}$ \\
\hline $1+$ & $\begin{array}{l}\text { Metaanálisis y revisiones sistemáticas bien realizados } \\
\text { de ensayos clínicos B }\end{array}$ \\
\hline $1-$ & $\begin{array}{l}\text { Metaanálisis, revisiones sistemáticas o ensayos } \\
\text { clínicos con alto riesgo de sesgo }\end{array}$ \\
\hline $2++$ & $\begin{array}{l}\text { Revisiones sistemáticas de alta calidad de estudios de } \\
\text { cohorte o de casos y controles; estudios de cohorte y } \\
\text { de casos y controles con muy bajo riesgo de sesgo y } \\
\text { con alta probabilidad de establecer relaciones causales }\end{array}$ \\
\hline $2+$ & $\begin{array}{l}\text { Estudios de cohorte y de casos y controles de buena } \\
\text { calidad con bajo riesgo de sesgo y probabilidad } \\
\text { moderada de establecer relaciones causales }\end{array}$ \\
\hline $2-$ & $\begin{array}{l}\text { Estudios de cohorte y de casos y controles con alto } \\
\text { riesgo de sesgo y probabilidad importante de que las } \\
\text { relaciones no sean causales }\end{array}$ \\
\hline 3 & $\begin{array}{l}\text { Estudios no analíticos, como reportes de casos y series } \\
\text { de casos }\end{array}$ \\
\hline Opinión del experto
\end{tabular}

\section{Revisiones sistemáticas}

Se realizaron dos revisiones sistemáticas: una sobre tratamiento y otra sobre diagnóstico de PP. Se sintetizó la información en tablas de evidencia. La metodología de estas revisiones sistemáticas se incluye en la síntesis de la evidencia ${ }^{4}$.

\section{Graduación de la evidencia}

La evidencia sobre intervenciones terapéuticas se graduó en ocho niveles con el sistema propuesto por la Scottish Intercollegiate Guidelines Network (SIGN) ${ }^{5}$ (Tabla 1), y la evidencia sobre estudios diagnósticos con el sistema propuesto por el Oxford Centre for Evidence-Based Medicine (OCEBM) ${ }^{6}$ (Tabla 2).

\section{Fuerza de la recomendación}

Las recomendaciones se formularon de acuerdo con lo propuesto en la metodología SIGN ${ }^{5}$ y pueden ser de dos tipos, fuertes o débiles, dependiendo de la ponderación de la fuerza de la evidencia, de la importancia y la magnitud de los desenlaces, y de las consideraciones sobre las preferencias de los pacientes. Las recomendaciones para las cuales no existe evidencia 
Tabla 2. Niveles de evidencia (OCEBM) ${ }^{5}$ para estudios sobre pruebas diagnósticas

\begin{tabular}{|c|c|}
\hline la & $\begin{array}{l}\text { Revisiones sistemáticas de estudios de nivel } 1 \\
\text { homogéneos }\end{array}$ \\
\hline Ib & Estudios de nivel 1 \\
\hline II & $\begin{array}{l}\text { Estudios de nivel } 2 \\
\text { Revisiones sistemáticas de estudios de nivel } 2\end{array}$ \\
\hline III & $\begin{array}{l}\text { Estudios de nivel } 3 \\
\text { Revisiones sistemáticas de estudios de nivel } 3\end{array}$ \\
\hline IV & $\begin{array}{l}\text { Consensos y opinión de expertos sin evaluación } \\
\text { crítica explícita }\end{array}$ \\
\hline $\begin{array}{l}\text { Estudios } \\
\text { de nivel } 1\end{array}$ & $\begin{array}{l}\text { Cumplen con los siguientes criterios: } \\
\text { - Comparación ciega con una prueba válida } \\
\text { como estándar de referencia } \\
\text { - Espectro de pacientes apropiado }\end{array}$ \\
\hline $\begin{array}{l}\text { Estudios } \\
\text { de nivel } 2\end{array}$ & $\begin{array}{l}\text { Tienen solo uno de los siguientes sesgos: } \\
\text { - Población no representativa } \\
\text { - Estándar de referencia imperfecto, o la } \\
\text { prueba que se evalúa es parte del estándar } \\
\text { de referencia, o el resultado de la prueba } \\
\text { índice afecta el desempeño del estándar de } \\
\text { referencia } \\
\text { - Comparación no cegada } \\
\text { - Estudios de casos y controles }\end{array}$ \\
\hline $\begin{array}{l}\text { Estudios } \\
\text { de nivel } 3\end{array}$ & $\begin{array}{l}\text { Tienen dos o más de los criterios enunciados } \\
\text { para los estudios de nivel } 2\end{array}$ \\
\hline
\end{tabular}

suficiente, pero que son prácticas comunes con base en la experiencia clínica y el consenso de los miembros del grupo elaborador de la GPC, se etiquetaron como «buena práctica clínica» (Tabla 3).

\section{Formulación y validación de recomendaciones}

Se conformaron grupos de trabajo de cuatro a cinco miembros para la elaboración de cada recomendación. Todas las recomendaciones fueron sometidas a consenso por los 24 miembros del grupo desarrollador de la GPC mediante el método Delphi. Después de cada ronda de discusión, los participantes registraron su voto y sus comentarios de manera anónima. A continuación, las recomendaciones se modificaron de acuerdo con los comentarios orales y escritos, y se sometieron a una siguiente ronda de discusión. El objetivo fue alcanzar la saturación de la discusión y un grado de acuerdo de más del $80 \%$ para cada recomendación.

Posteriormente, se invitó a participar a los endocrinólogos pediatras certificados que acudieron al XX
Congreso Anual de la Sociedad Mexicana de Endocrinología Pediátrica a responder un cuestionario en línea, para conocer el grado de acuerdo para cada una de las recomendaciones.

\section{Resultados}

Se generaron 43 recomendaciones en total: 12 recomendaciones sobre el diagnóstico de $\mathrm{PP}^{7}$, siete sobre el diagnóstico de causas secundarias ${ }^{8}$, ocho sobre intervenciones para la inhibición de la pubertad ${ }^{9}$, cinco sobre otras intervenciones en $\mathrm{PP}^{10}$ y 14 para la monitorización del tratamiento y el seguimiento de estos pacientes $^{11}$. Las recomendaciones y su sustento se detallan en el resto de los siguientes artículos de este suplemento, así como las tablas de evidencia de las tres revisiones sistemáticas que se realizaron.

Se efectuaron tres rondas de discusión y validación de cada una de las recomendaciones, en las que participaron los 24 miembros del grupo desarrollador de la GPC. Después de la tercera ronda, no se registraron comentarios novedosos que quedaran sin discutir y se alcanzó el consenso general para todas las recomendaciones.

Además, 143 endocrinólogos pediatras certificados respondieron el cuestionario de validación en línea. Se obtuvieron, al menos, 123 respuestas válidas para cada una de las recomendaciones y un porcentaje de acuerdo mayor del $90 \%$ para todas ellas.

\section{Glosario de los términos utilizados en la guía de práctica clínica}

Adrenarca: activación de la secreción adrenal de andrógenos.

Análogos de GnRH (aGnRH) de depósito: sintéticos similares al decapéptido de GnRH que, tras la estimulación inicial, suprimen la liberación pulsátil de hormona luteinizante (LH) y de hormona estimulante del folículo (FSH) mediante la desensibilización de los gonadotropos a la GnRH. Gracias a la sustitución de aminoácidos 0 de alteraciones hidrófobas, son más potentes y poseen una duración de acción mayor que la $\mathrm{GnRH}$, de semanas o meses.

Caracteres sexuales secundarios: en el sexo femenino, crecimiento mamario, ensanchamiento de cadera, crecimiento de vello púbico y axilar; en el sexo masculino, crecimiento de vello facial, axilar y púbico, y crecimiento laríngeo.

Edad ósea: método de evaluación del estado de maduración esquelética mediante radiografía simple. 
Tabla 3. Forma de las recomendaciones

\section{Juicio}

Las consecuencias deseables de la intervención claramente superan las consecuencias indeseables en CASI TODOS los pacientes. Implicación: los clínicos deben seguir una recomendación fuerte a menos que haya una razón clara e importante para un abordaje alternativo

Las consecuencias deseables probablemente superan las consecuencias indeseables en la MAYORÍA de los pacientes. La elección de la intervención es más susceptible de variar dependiendo de las preferencias y de los valores personales de cada paciente, por lo que el profesional deberá invertir más tiempo discutiendo las diferentes opciones con el paciente

El balance entre las consecuencias deseables e indeseables no es claro

Recomendación basada solo en la experiencia clínica del grupo desarrollador de la guía de práctica clínica
Fuerza de la recomendación

Recomendación fuerte

Recomendación condicional

Recomendación para la investigación y posiblemente una recomendación condicional restringida a protocolos de investigación

Buena práctica clínica
Escape del bloqueo con aGnRH: falla en el bloqueo del eje hipotálamo-hipófisis-gónada por aGnRH.

Estadios de desarrollo mamario de Tanner: sistema de evaluación clínica del grado de desarrollo puberal en el sexo femenino (Tabla 4$)^{12}$.

FSH: hormona estimulante del folículo.

GnRH: hormona liberadora de gonadotropina.

LH: hormona luteinizante.

Pronóstico de talla: talla final estimada a partir de la talla y de otros datos clínicos del presente mediante alguno de los métodos desarrollados con este fin.

Prueba de estimulación con aGnRH: prueba dinámica para evaluar el estado de activación puberal. Consiste en la medición de la LH tras la administración de GnRH o algún análogo de acción rápida. La elevación de la LH más allá del punto de corte se interpreta como evidencia de activación puberal hipotálamo-hipofisiaria. Los puntos de corte para la concentración de LH y el tiempo tras el estímulo en el que se mide varían según el protocolo. En algunos protocolos también se miden las concentraciones de FSH y de hormonas sexuales.

Pseudopubertad precoz o pubertad precoz periférica: aparición de caracteres sexuales secundarios antes de los 8 años en el sexo femenino o de los 9 años en el sexo masculino como resultado de un proceso distinto de la activación del eje hipotálamo-hipófisis-gónada.

Pubarca: aparición de vello púbico.

Pubertad precoz: crecimiento de las glándulas mamarias en niñas menores de 8 años o del volumen testicular $\left(>4 \mathrm{~cm}^{3}\right)$ en niños menores de 9 años, desencadenado por el aumento en la producción hipofisiaria de gonadotropinas.
Tabla 4. Estadios de desarrollo mamario según Marshall y Tanner 6

I Prepuberal: solo existe elevación del pezón

II Botón mamario: elevación de la mama y del pezón como un pequeño montículo, con aumento del diámetro areolar

III Mayor aumento del tamaño de la mama y la aréola, sin separación de sus contornos

IV Proyección de la aréola y del pezón hasta formar un montículo secundario por encima del nivel de la mama

V Nivel maduro: proyección solo del pezón

Pubertad precoz idiopática: pubertad precoz en la que después de una búsqueda intencionada no se logra identificar la etiología.

Pubertad precoz secundaria: maduración del eje hipotálamo-hipófisis-gónada como resultado de una causa específica (por ejemplo, lesión intracraneal, exposición a esteroides sexuales, entre otros).

Salud ósea: estado de la arquitectura y de la mineralización del hueso que minimiza el riesgo de fracturas.

Talla adulta: talla alcanzada cuando el crecimiento se ha completado.

Talla casi adulta: talla alcanzada en un estadio avanzado de la pubertad, pero antes de que se hayan cerrado todos los cartílagos de crecimiento.

Telarca: desarrollo del tejido mamario a partir de la forma infantil (Tanner II) como resultado de la estimulación estrogénica en el sexo femenino. 


\section{Conflicto de intereses}

Laboratorio Asofarma México brindó apoyo logístico para las reuniones del grupo elaborador de la GPC, y apoyo para la edición y publicación del documento.

\section{Bibliografía}

1. Sun SS, Schubert CM, Chumlea WC, Roche AF, Kulin HE, Lee PA, et al National estimates of the timing of sexual maturation and racial differences among US children. Pediatrics. 2002;110:911-9.

2. Aksglaede L, Sorensen K, Petersen JH, Skakkebaek NE, Juul A. Recent decline in age at breast development: The Copenhagen Puberty Study. Pediatrics. 2009;123:e932-9.

3. Herman-Giddens ME, Steffes J, Harris D, Slora E, Hussey M, Dowshen SA et al. Secondary sexual characteristics in boys: data from the Pediatric Research in Office Settings Network. Pediatrics. 2012;130:e1058-68.

4. Castilla-Peón MF, Torres-Tamayo M, Zurita-Cruz JN, Aguilar-Herrera BE, Miranda-Lora AL, Rivera-Hernández AJ, et al. Síntesis de la evidencia: Guía de práctica clínica para el diagnóstico y el tratamiento de la pubertad precoz. Bol Med Hosp Infant Mex. 2020;77(Supl 1):35-40.

5. Scottish Intercollegiate Guidelines Network; Healthcare Improvement Scotland. SIGN 50: a guideline developer's handbook. Checklists. Edinburgh; 2015. Disponible en: sign.ac.uk.
6. Oxford Centre for Evidence-Based Medicine (CEBM). OCEBM levels of evidence. (Actualizado el 1 de mayo de 2016.) Disponible en: cebm.net.

7. Miranda-Lora AL, Torres-Tamayo M, Zurita-Cruz JN, Aguilar-Herrera BE, Calzada-León R, Rivera-Hernández AJ, et al. Diagnóstico de pubertad precoz: Guía de práctica clínica para el diagnóstico y el tratamiento de la pubertad precoz. Bol Med Hosp Infant Mex. 2020;77(Supl 1):7-14.

8. Torres-Tamayo M, Calzada-León R, Rivera-Hernández AJ, Zurita-Cruz JN, Aguilar-Herrera BE, Miranda-Lora AL, et al. Diagnóstico de causas secundarias de pubertad precoz: Guía de práctica clínica para el diagnóstico y el tratamiento de la pubertad precoz. Bol Med Hosp Infant Mex. 2020;77(Supl 1):15-8.

9. Torres-Tamayo M, Zurita-Cruz JM, Aguilar-Herrera BE, Miranda-Lora AL, Calzada-León R, Rivera-Hernández AJ, et al. Inhibición de la pubertad: Guía de práctica clínica para el diagnóstico y el tratamiento de la pubertad precoz. Bol Med Hosp Infant Mex. 2020;77(Supl 1):19-25.

10. Torres-Tamayo M, Zurita-Cruz JN, Aguilar-Herrera BE, Miranda-Lora AL, Calzada-León R, Rivera-Hernández AJ, et al. Intervenciones adyuvantes en el manejo de la pubertad precoz: Guía de práctica clínica para el diagnóstico y el tratamiento de la pubertad precoz. Bol Med Hosp Infant Mex. 2020;77(Supl 1):26-8.

11. Zurita-Cruz JN, Torres-Tamayo M, Aguilar-Herrera BE, Miranda-Lora AL Rivera-Hernández AJ, Calzada-León R, et al. Monitorización durante el tratamiento de la pubertad precoz: Guía de práctica clínica para el diagnóstico y el tratamiento de la pubertad precoz. Bol Med Hosp Infant Mex. 2020;77(Supl 1):29-34.

12. Marshall WA, Tanner JM. Variations in pattern of pubertal changes in girls. Arch Dis Child. 1969;44:291-303. 